\title{
Morphological study of magnesium hydroxide nanoparticles precipitated in dilute aqueous solution
}

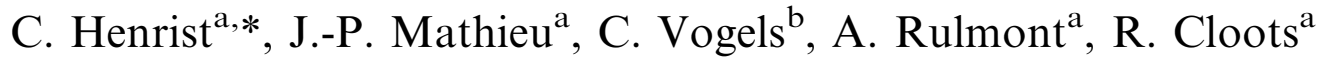 \\ ${ }^{a}$ Laboratory of Inorganic Structural Chemistry, University of Liège, B6 Sart Tilman, B-4000 Liège, Belgium \\ ${ }^{\mathrm{b}}$ Solvay S.A., B-1120 Brussels, Belgium
}

Received 6 September 2002; accepted 11 October 2002

Communicated by D.T.J. Hurle

\begin{abstract}
Among other applications, magnesium hydroxide is commonly used as a flame-retardant filler in composite materials, as well as a precursor for magnesium oxide refractory ceramic. The microstructure of the powder is of prime importance in both technical applications. The influence of synthesis parameters on the morphological characteristics of magnesium hydroxide nanoparticles precipitated in dilute aqueous medium was studied. Several parameters were envisaged such as chemical nature of the base precipitant, type of counter-ion, temperature and hydrothermal treatment. Special attention was given to the obtaining of platelet-shaped, nanometric and de-agglomerated powders. The powders were characterized in terms of particle size distribution, crystal habits, morphology and ability to be redispersed in water. X-ray diffraction (XRD), scanning electron microscopy (SEM), transmission electron microscopy (TEM), nitrogen adsorption and laser diffusion analyses were used for this purpose.
\end{abstract}

(C) 2002 Elsevier Science B.V. All rights reserved.

Keywords: A1. Crystal morphology; A1. X-ray diffraction; A2. Growth from solutions; A2. Hydrothermal crystal growth; B1. Magnesium hydroxide

\section{Introduction}

The precipitation of magnesium hydroxide is of great interest in the context of the manufacture of magnesium oxide from magnesium chloride contained in seawater [1,2] or natural brines [2]. When magnesium hydroxide powder is used as a precursor for magnesium oxide synthesis, its

\footnotetext{
*Corresponding author. Tel.: +32-4-366-3417; fax: +32-4366-3413.

E-mail address: catherine.henrist@ulg.ac.be (C. Henrist).
}

particle size, shape and agglomeration level constitute key parameters in the sintering step and the processing of the final ceramics.

Inorganic hydrates are commonly used in flameretardant composite formulations due to their ability to undergo endothermic dehydration in fire conditions [3-7]. There is a growing interest in magnesium hydroxide as a flame retardant filler that does not evolve toxic and corrosive substances upon combustion. Moreover, magnesium hydroxide can be used at higher processing temperatures than the most widely used aluminium trihydrate 
fillers. It has to be noticed that the inorganic filler can affect the mechanical properties of the polymer matrix. The mechanical strength of composites can be enhanced by dispersing nanometer sized inorganic platelets of the filler in the polymer.

A nanocomposite is characterized by a high degree of dispersion of an inorganic filler, to a nanoscopic level, in a polymer matrix. Many studies have been recently focusing on the development and characterization of new nanocomposites exhibiting anisotropic morphologies [8-14]. Specific mechanical and physical properties are expected when the filler particles are plateletshaped $[15,16]$. The advantage of composites containing nanoplatelets is mainly the improvement of their mechanical reinforcement properties as well as "barrier properties" that are supposed to play a significant role in fire resistance, for lower filler loading than traditional composites [14]. The combination of these two characteristics (morphology and water release), regarding magnesium hydroxide, renders it a good candidate for fire resistance applications.

Crystalline magnesium hydroxide, the so-called Brucite [17-19], belongs to the bivalent metal hydroxides group, whose crystal structure is a layered $\mathrm{CdI}_{2}$-type arrangement. Successive hexagonal $\mathrm{Mg}^{2+}$ ions layers and $\mathrm{OH}^{-}$ions layers are stacked one upon another. The magnesium cation is six-fold coordinated by hydroxyl groups, thus forming $\mathrm{Mg}(\mathrm{OH})_{6}$ octahedra. Complete crystal structure descriptions can be found in the specialized literature [17,19-21]. Such a layered crystal structure is an advantage for platelet-shaped crystallization of the compound.

Magnesium hydroxide can be obtained by several methods $[17,21,22]$, i.e. hydration of magnesium oxide $\mathrm{MgO}$, [22,23], precipitation of a magnesium salt with an alkaline solution [24-27], electrolysis of an aqueous magnesium salt solution. Industrial production of magnesium hydroxide is mainly driven by the two first methods [2830]. The objective of this paper is to investigate how to influence the magnesium hydroxide particle size, shape and agglomeration level, during and after synthesis by alkaline precipitation in dilute aqueous medium.

\section{Experimental procedure}

The samples were obtained by precipitation, at a controlled temperature, of a magnesium salt solution of concentration $0.75 \mathrm{~mol} / 1$ by addition of an alkaline solution $\left(\mathrm{NaOH}\right.$ or $\left.\mathrm{NH}_{4} \mathrm{OH}\right)$ of concentration $1.5 \mathrm{~mol} / 1$. Both reactants were simultaneously added into an ultrasonicated bath following the controlled-double jet precipitation technique, with the help of peristaltic pumps working at a discharge of $3 \mathrm{ml} / \mathrm{min}$. A $450 \mathrm{ml}$ volume of alkaline water $\left[\mathrm{pH} 10\right.$, either $\mathrm{NH}_{4} \mathrm{OH}$ or $\mathrm{NaOH}]$ initially present in the reactor, vigorous stirring was applied during the addition of the reactants, as well as during the ageing of the precipitate in the mother liquor. The suspension was allowed to age at the synthesis temperature for one day, then subsequently at room temperature for 2 days. The solid phase obtained was recovered by centrifugation at $3500 \mathrm{rpm}$ and washed twice with slightly alkaline water, then air-dried at $60^{\circ} \mathrm{C}$ overnight for powders characterization, or redispersed in distilled water for measurements in aqueous suspension. Philips et al. [31] showed that the very brief process of washing with distilled water caused no significant change in particle size or shape, due to the very low solubility of magnesium hydroxide. Some of the samples were submitted to a hydrothermal treatment: the mother liquor was placed in a closed Teflon bottle in an oven pre-heated at $170^{\circ} \mathrm{C}$ during $14 \mathrm{~h}$ to 7 days. The composition and reaction conditions of the samples are given in Table 1 .

The particle morphology was observed by scanning electron microscopy (SEM) on a Philips ESEM XL30 FEG microscope after coating the

Table 1

Composition and reaction conditions of the different samples

\begin{tabular}{llll}
\hline Sample & $\mathrm{Mg} \mathrm{source}$ & Base source & Temperature $\left({ }^{\circ} \mathrm{C}\right)$ \\
\hline 1 & $\mathrm{MgCl}_{2}$ & $\mathrm{NaOH}$ & 60 \\
2 & $\mathrm{MgCl}_{2}$ & $\mathrm{NH}_{4} \mathrm{OH}$ & 60 \\
3 & $\mathrm{MgCl}_{2}$ & $\mathrm{NH}_{4} \mathrm{OH}$ & 25 \\
4 & ${\mathrm{Mg}\left(\mathrm{NO}_{3}\right)_{2}}$ & $\mathrm{NH}_{4} \mathrm{OH}$ & 25 \\
5 & $\mathrm{MgSO}_{4}$ & $\mathrm{NH}_{4} \mathrm{OH}$ & 25 \\
6 & $\mathrm{MgCl}_{2}$ & $\mathrm{NH}_{4} \mathrm{OH}$ & 10 \\
7 & $\mathrm{MgCl}_{2}$ & $\mathrm{NH}_{4} \mathrm{OH}$ & 47 \\
\hline
\end{tabular}


sample with a very thin gold layer deposited by sputtering under vacuum. Observation of the particles was also made by transmission electron microscopy (Philips CM100 TEM working with a $100 \mathrm{kV}$ acceleration voltage) on powders simply deposited onto a carbon-coated copper grid. Crystallinity of the product was checked by Xray diffraction (XRD) on a Siemens D5000 powder diffractometer working with $\mathrm{Cu} \mathrm{K}_{\alpha}$ radiation and $\mathrm{Ni}$ filter. Particles size distribution in solution was measured with a Malvern Mastersizer Hydro2000S granulometer, while the mean size of the particles in the dry powder was determined by visual analysis of SEM micrographs, on a large number of particles (ca. 200). Anionic dispersant used for water dispersion characterization, Dispex $\mathrm{N} 40 \mathrm{~V}$, was purchased from Ciba Specialty Chemicals Co. and used as received. The specific surface measurements were carried out on a Monosorb® surface area analyser from Quantachrome working with the single point method, which gives directly access to the value of the surface area with minor error, of the order of $5 \%$. The system uses a $\mathrm{N}_{2} / \mathrm{He}$ flow with $30 \mathrm{~mol}^{\%}$ of nitrogen.

\section{Results and discussion}

Several reaction parameters were envisaged in this study: the chemical nature of the base precipitant (samples 1,2), the temperature during addition (samples 2,3,6,7), the chemical nature of the counter-ion in the magnesium salt (samples 3,4,5), and additional hydrothermal treatment.

\subsection{Influence of the nature of the base precipitant}

\subsection{1. $\mathrm{MgCl}_{2}+\mathrm{NaOH}$ at $60^{\circ} \mathrm{C}$.}

The precipitation reaction taking place in this case is

$$
\mathrm{MgCl}_{2}+2 \mathrm{NaOH} \rightarrow \mathrm{Mg}(\mathrm{OH})_{2}+2 \mathrm{NaCl} .
$$

As can be seen in Fig. 1a, the result of the reaction between magnesium chloride and sodium hydroxide, driven as described before at a temperature of $60^{\circ} \mathrm{C}$, is somewhat surprising. To our knowledge, the globular microstructure of the magnesium hydroxide particles obtained here has never been reported, due to the natural tendency of this compound to form hexagonal platelets, which is related to its crystal structure. This globular structure can be described as large aggregates of spherical units which have a diameter $\sim 300 \mathrm{~nm}$, themselves being made of spherical smaller sub-units, and so on. Several parameters were varied while keeping the sodium hydroxide as the precipitant base: ageing time at $60^{\circ} \mathrm{C}$ (up to 1 month), discharge value of the reactant solutions into the reactor, temperature of precipitation $\left(25^{\circ} \mathrm{C}\right)$, concentration of the reactant solutions. Invariably, this globular-type superstructure was observed. Even a hydrothermal treatment at $180^{\circ} \mathrm{C}$ for $14 \mathrm{~h}$ did not change the morphology of the particles.

A precise mechanistic interpretation is beyond the scope of this work, but it can be postulated that the $\mathrm{pH}$ of the solution, and the chemical nature of ions present in solution, play a crucial role during crystal growth. Several authors [31,32] have determined the isoelectric point of magnesium hydroxide particles in water, which is situated at ca. $\mathrm{pH}$ 12. In the case the synthesis with $\mathrm{NaOH}$ used as the base, the $\mathrm{pH}$ reached in the reacting mixture during synthesis is higher than 13. Therefore, the net residual electric charge on the surface of the particles is expected to be negative. The elevated $\mathrm{pH}$ value also creates a high supersaturation level due to the large concentration of hydroxyl ions in solution. This results in an extremely fast nucleation process generating tiny, not well-defined nuclei. Following the nucleation step, cations in solution migrate towards the growing crystals. Due to their high concentration and small hydration sphere in solution, the sodium ions can probably adsorb significantly onto all the facets of the nuclei, without any selectivity, thus hindering the income of fresh magnesium ions and subsequent growth. Those very small and isotropic particles tend to aggregate in order to lower their surface energy, finally adopting the globular-type structure reported in Fig. 1a. This agglomeration step is further enhanced by the elevated temperature conditions. 

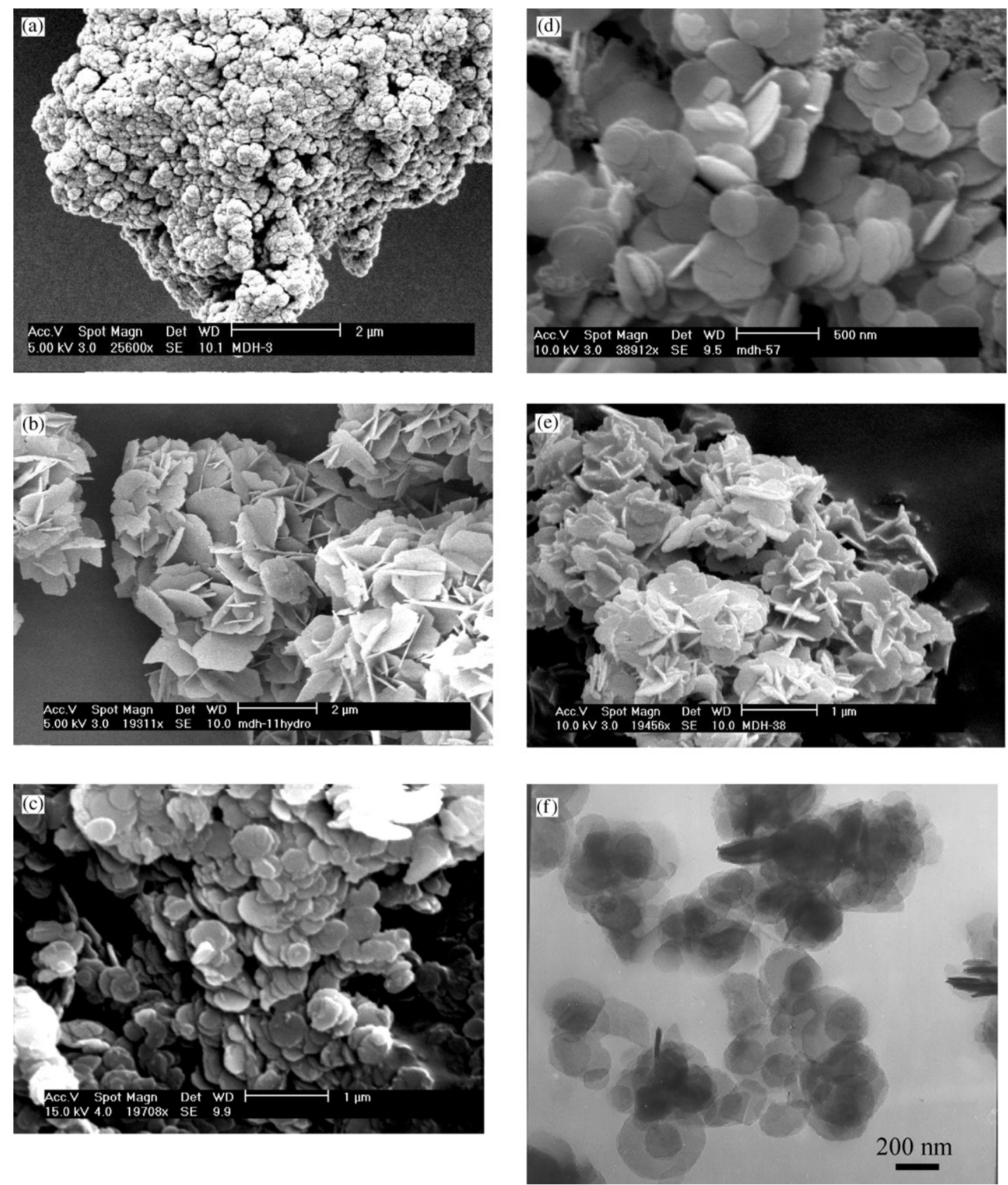

Fig. 1. SEM micrographs of (a) sample 1, (b) sample 2, (c) sample 3, (d) sample 4, (e) sample 5, (f) TEM micrograph of sample 3.

\subsection{2. $\mathrm{MgCl}_{2}+\mathrm{NH}_{4} \mathrm{OH}$ at $60^{\circ} \mathrm{C}$.}

The precipitation reaction taking place in this case is

$\mathrm{MgCl}_{2}+2 \mathrm{NH}_{4} \mathrm{OH} \rightarrow \mathrm{Mg}(\mathrm{OH})_{2}+2 \mathrm{NH}_{4} \mathrm{Cl}$
The morphology of the particles obtained in the presence of ammonia at $60^{\circ} \mathrm{C}$ is shown in Fig. 1 b. As can be seen on the SEM micrograph, a plateletlike morphology was attained, but exhibits a peculiar arrangement typically resulting from 
intergrowth mechanism. It is clearly observed that the individual platelets are not simply randomly piled up, but have simultaneously grown from preagglomerated seeds and are therefore impossible to separate from each other by any soft process. Vigorous grinding or hydrothermal post-treatment of the powder does not affect this particle morphology obtained at $60^{\circ} \mathrm{C}$.

The $\mathrm{pH}$ of the suspension when using $\mathrm{NH}_{4} \mathrm{OH}$ as the base precipitant was lower than that obtained by using $\mathrm{NaOH}$, at ca. $\mathrm{pH} 10$. According to the isoelectric point value, the particles are positively charged and the adsorption of cations is not favoured anymore. Moreover, the ammonium cation is larger than the sodium ion and is not easily adsorbed on the crystal facets. Adsorption of hydroxyl ions onto the basal plane of the crystallites is thought to promote the edgewise growth of the particles. Since the initial water volume is set at $\mathrm{pH} 10$, hydroxyl groups are in slight excess during the whole process. Due to a high supersaturation level caused by this excess of hydroxyl ions, a fast nucleation process takes place leading to agglomerates. Since the solubility of magnesium hydroxide decreases when increasing the temperature [33], this supersaturation state is further enhanced by the high temperature conditions. The subsequent growth process is not inhibited in these conditions and gives rise to some edgewise crystal growth. This special aggregation mode exists in nature and gives rise to concretions with similar morphology [34].

\subsection{Effect of temperature}

As partly described before, performing the addition at an elevated temperature exerts a strong influence on the resulting morphology and aggregation behaviour of the magnesium hydroxide particles (see Fig. 1b). For the study of temperature dependence, all syntheses were driven starting from aqueous ammonia and magnesium chloride. Decreasing the synthesis temperature from $60^{\circ} \mathrm{C}$ to $25^{\circ} \mathrm{C}$ shows a favourable effect on the desired morphology of the particles, as can be seen in Fig. 1c and f. No intergrowth of the particles is observed anymore. It appears that the agglomeration of the primary nuclei is not favoured at this temperature. Small platelet-shaped particles with a slight tendency towards the hexagonal morphology are observed after ageing. On a morphological point of view, all the synthesis made below the temperature of $60^{\circ} \mathrm{C}$ gives rise to the same thin, platelet-shaped particles with a more or less circular contour, as displayed in Fig. 1c, while the synthesis at $60^{\circ} \mathrm{C}$ produces the typical "sand rose" feature displayed in Fig. 1b. The XRD pattern also confirms this difference. It can be seen in Fig. 3 that sample 3 exhibits a higher $I_{001} / I_{110}$ ratio than sample 2 , which indicates a more pronounced orientation of the single platelets towards the incident $\mathrm{X}$-ray radiation. This preferential orientation is not possible in the case of "sand rose" morphology. Concerning the mean size of the particles, two characterization techniques were used and gave different results: laser diffusion and image analysis. When measured with laser diffusion on a suspension in water (Fig. 2), the mean size of equivalent volume sphere ${ }^{1}$ is more or less identical for the syntheses driven at $10^{\circ} \mathrm{C}$, $25^{\circ} \mathrm{C}$ and $47^{\circ} \mathrm{C}$ and is ca. $220 \mathrm{~nm}$, which corresponds to single de-agglomerated particles. Few larger aggregates can be observed. The sample synthesized at $60^{\circ} \mathrm{C}$ exhibits a larger mean particles size, ca. $450 \mathrm{~nm}$, due to the stronger cohesion of the aggregates. When measured by image analysis on the basis of SEM micrographs taken on the dry powder, size variations can be observed, as shown in Table 2. Working at low temperature favours a slow nucleation process and growth of the crystallites, which can lead to larger but fewer particles, when all the precursor species in solution have been incorporated into the solid phase. This is illustrated when comparing samples 3 and 7 : increasing the synthesis temperature from $25^{\circ} \mathrm{C}$ to $47^{\circ} \mathrm{C}$ results in an overall decrease of the mean particle diameter, from 360 to $218 \mathrm{~nm}$. The sample synthesized at $10^{\circ} \mathrm{C}$ was aged at $2^{\circ} \mathrm{C}$ in a water-ice thermostat. Since it exhibits a smaller particle size than expected, we suggest that at this

\footnotetext{
${ }^{1}$ The results of laser size are expressed in terms of equivalent sphere. The instrument uses the volume of the particle to measure its size: it calculates the diameter of an imaginary sphere that is equivalent in volume. Consequently, in the case of platelets, this value does not represent the actual particle diameter.
} 


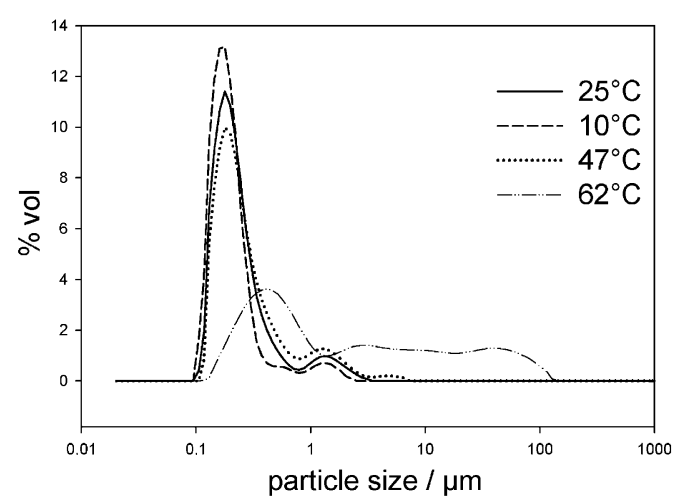

Fig. 2. Particle size distribution in water: effect of synthesis temperature.

Table 2

Mean particle size determined by image analysis

\begin{tabular}{lll}
\hline Sample & Temperature $\left({ }^{\circ} \mathrm{C}\right)$ & Size $(\mathrm{nm})$ \\
\hline 6 & 10 & 341 \\
3 & 25 & 360 \\
7 & 47 & 218 \\
2 & 60 & 302 \\
\hline
\end{tabular}

very low temperature, the system was not given enough time to reach its equilibrium state and therefore, the growing particles have not reached their final size. The data for $60^{\circ} \mathrm{C}$ cannot be interpreted in the same way due to the difficulty to distinguish single platelets diameter in the "sand rose" morphology. In conclusion, it can be said that the synthesis has to be driven between $25^{\circ} \mathrm{C}$ and $50^{\circ} \mathrm{C}$ in order to get single platelets within a reasonable ageing time (Figs. 2 and 3).

\subsection{Influence of the magnesium source at $25^{\circ} \mathrm{C}$}

For the study of the influence of the counter-ion in the magnesium salt, the synthesis was always performed at $25^{\circ} \mathrm{C}$ with ammonia as the base precipitant. As said before, the $\mathrm{pH}$ value was lower than 12 and the particles in suspension are therefore expected to be positively charged.

\subsection{1. $\mathrm{MgCl}_{2}+\mathrm{NH}_{4} \mathrm{OH}$.}

These synthesis conditions correspond to sample 3, which is described earlier in the study of

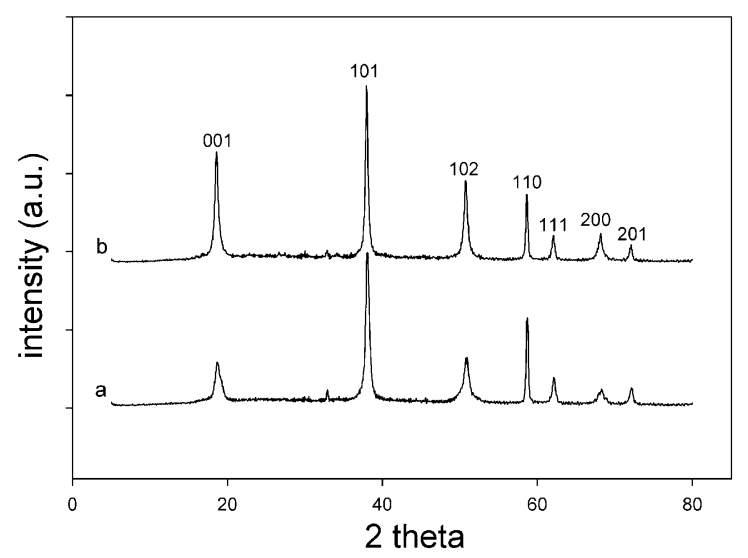

Fig. 3. XRD pattern of (a) sample 2, $I_{001} / I_{110}=0.51$ and (b) sample $3, I_{001} / I_{110}=1.6$.

temperature dependence. Corresponding SEM and TEM micrographs are displayed in Fig. 1c and $\mathrm{f}$, respectively, and show de-agglomerated, nearly circular platelets with a mean diameter of ca. $360 \mathrm{~nm}$.

\subsection{2. $\mathrm{Mg}\left(\mathrm{NO}_{3}\right)_{2}+\mathrm{NH}_{4} \mathrm{OH}$}

Aqueous magnesium nitrate was reacted with aqueous ammonia, all other conditions being similar to the synthesis starting from $\mathrm{MgCl}_{2}$, in order to study the influence of the presence of an oxo-anion on the resulting morphology. The reaction taking place is the following:

$$
\begin{aligned}
& \mathrm{Mg}\left(\mathrm{NO}_{3}\right)_{2}+2 \mathrm{NH}_{4} \mathrm{OH} \\
& \quad \rightarrow \mathrm{Mg}(\mathrm{OH})_{2}+2 \mathrm{NH}_{4} \mathrm{NO}_{3} .
\end{aligned}
$$

The nitrate ions do not influence the $\mathrm{pH}$ of the solution, which is mainly determined by the ammonia solution during addition. The morphology of the particles obtained after synthesis and ageing, observed by SEM, is displayed in Fig. 1d. A platelet shape but a nearly circular contour characterizes the particles. Nevertheless, no significant morphological difference with the chloride-based synthesis can be deduced from these observations. The mean diameter was determined to be $351 \mathrm{~nm}$, which is close to the value estimated for the chloride-based synthesis. In conclusion, starting from magnesium nitrate does not promote any improvement nor deterioration on a morphological point of view, but can still be 
envisaged if the chloride has to be avoided for any reason.

\subsection{3. $\mathrm{MgSO}_{4}+\mathrm{NH}_{4} \mathrm{OH}$}

The reaction taking place is the following:

$\mathrm{MgSO}_{4}+2 \mathrm{NH}_{4} \mathrm{OH} \rightarrow \mathrm{Mg}(\mathrm{OH})_{2}+\left(\mathrm{NH}_{4}\right)_{2} \mathrm{SO}_{4}$.

Using magnesium sulphate as the magnesium source leads to agglomerated particles, as shown in Fig. 1e. Moreover, it is likely that an intergrowth phenomenon has taken place, leading to randomly tangled particles connected to each other. This feature is similar to that obtained for sample 2 $\left(\mathrm{MgCl}_{2}+\mathrm{NH}_{4} \mathrm{OH}\right.$ at $\left.60^{\circ} \mathrm{C}\right)$, except that the mean thickness of the sub-units seems to be slightly larger. It is therefore suspected that the presence of sulphate ions increases the supersaturation level, probably due to its weak base property. A sudden generation of nuclei's in the medium and their agglomeration is unavoidable. Again in this case, no soft process can separate the particles.

\subsection{Effect of hydrothermal treatment}

Hydrothermal conditions are encountered when water is maintained in a closed volume at a temperature higher than $100^{\circ} \mathrm{C}$. The reactivity of the species in solution is greatly influenced by three properties of the water as a solvent: (i) the dielectric constant, which decreases as temperature rises; (ii) the viscosity, which decreases when temperature increases and (iii) the dissociation constant of the water, which is higher at elevated temperature. These modifications favour electrostatic interactions, lower kinetic barriers and accelerate the ripening processes [35]. Therefore, it is not surprising to observe great improvements in the morphology and crystallinity of a magnesium hydroxide sample submitted to a hydrothermal treatment. Fig. 6 shows the particles from sample 3 observed by SEM after a 1-week hydrothermal treatment at $170^{\circ} \mathrm{C}$. It can be seen that larger, well-defined hexagonal platelets are formed. This enlargement of the crystallites was also verified by nitrogen adsorption measurements, as displayed in Fig. 4: the specific surface area of the dried powder gradually decreases with the duration of hydrothermal treatment, while the mean particle size, determined by SEM image analysis in this case, is getting larger. It can be observed that both curves are symmetric with respect to each other, and that the major evolution in size and specific surface is accomplished within the first 2 days of hydrothermal treatment. Further treatment in the autoclave only promotes a few changes in these properties. Fig. 5 displays the XRD patterns of the sample before treatment (a) and after a 7-days stay in the autoclave (b). An overall enhancement of the crystallinity is clearly visible since intensity of the diffraction peaks is increased to a large extent in curve (b). Moreover, comparing the intensity ratio between reflections $\left[\begin{array}{lll}0 & 0\end{array}\right]$ and $\left[\begin{array}{lll}1 & 1 & 0\end{array}\right]$ gives a possible indication of a

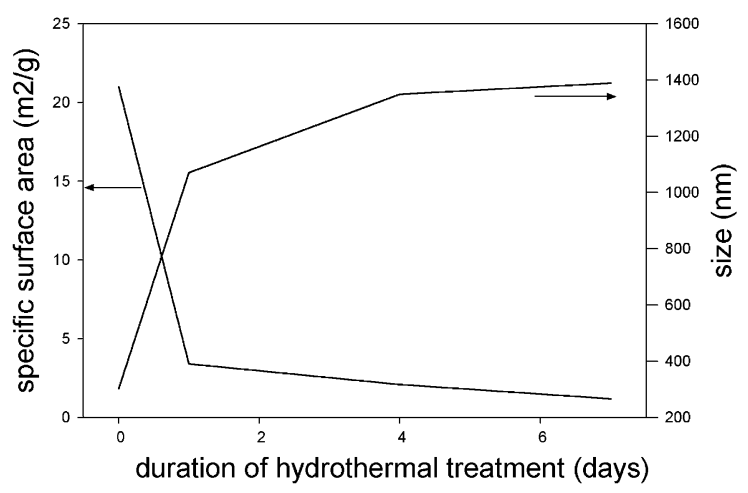

Fig. 4. Effect of hydrothermal treatment duration (in days) on particles size and specific surface area of the powder.

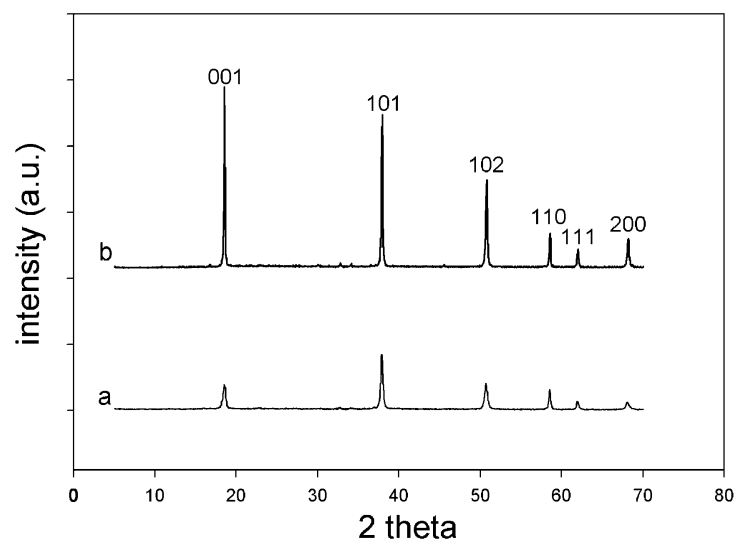

Fig. 5. XRD pattern of sample 3. Curve a: untreated, $I_{001} / I_{110}=1.26$. Curve b: after 7 days of hydrothermal treatment, $I_{001} / I_{110}=5.02$. 
more pronounced orientation effect in the case of the curve (b), due to particles exhibiting a larger aspect ratio.

\subsection{Dispersability}

Aqueous slurries of magnesium hydroxide can be used to incorporate the inorganic filler in a water-soluble polymer, or can even be added during the synthesis of an emulsion-driven polymerization of PVC, for example. In this respect, it is interesting to control the stability of the suspension and avoid the formation of large agglomerates of particles in water, prior to the mixing with the polymer. In Fig. 7 short dash

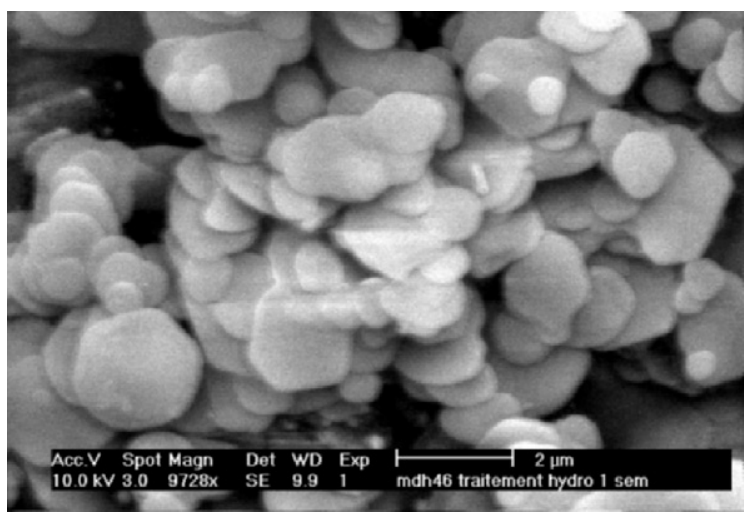

Fig. 6. Sample 3 after 7 days of hydrothermal treatment at $170^{\circ} \mathrm{C}$. curve shows the size distribution in volume of the magnesium hydroxide powder corresponding to sample 3 (Figs. 6 and 7), without any dispersing aid other than stirring. It shows that the platelets tend to aggregate in water to form big clusters of mean size ca. $9 \mu \mathrm{m}$. Applying a $30 \mathrm{~min}$ ultrasonication at $100 \mathrm{~mW}$ to the suspension does not change the situation. A dispersant additive was then used in order to improve the dispersion of the particles in suspension: Dispex $N 40 \mathrm{~V}$, a sodium polyacrylate in aqueous solution. Five drops of Dispex $N 40 \mathrm{~V}$ solution $(0.1445 \mathrm{~g})$ were added to the tank containing $10 \mathrm{ml}$ of mother $\mathrm{MDH}$ solution and $110 \mathrm{ml}$ of water. Stirring was applied during $20 \mathrm{~min}$ before making the granulometric measurement (Fig. 7-long dash curve). A widening of the distribution for smaller particles appears, as expected from the addition of a good dispersant, but the distribution is still too large and the particle separation is not complete. By exposing the solution to ultrasound during $15 \mathrm{~min}$, the distribution curve (Fig. 7-solid line) shows an important maximum at ca. $0.18 \mu \mathrm{m}$ which can be assigned to single platelets in suspension. Another less prominent maximum is also observed at ca. $1.3 \mu \mathrm{m}$. Increasing the Dispex $N 40 \mathrm{~V}$ concentration does not improve the dispersion effect. It is therefore likely that Dispex $N 40 \mathrm{~V}$ acts rather as a stabiliser of particles previously mechanically separated by the ultrasonic bath. On the other hand, the particle dispersion cannot be performed only by ultrasonic exposure. It is important to

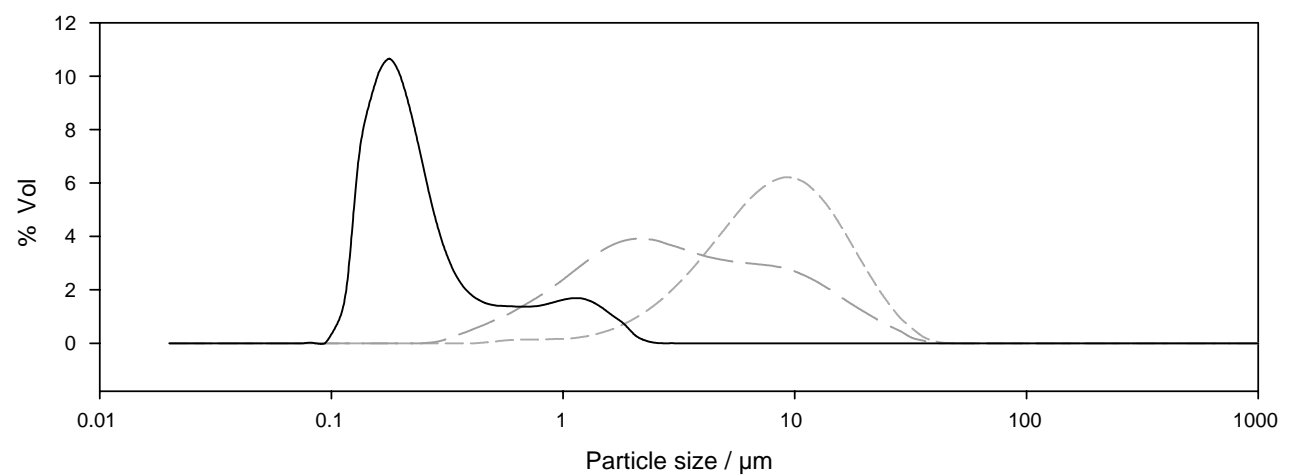

Fig. 7. Particle size distribution in volume \% for sample 3. Short dash: mother liquor; Long dash: mother liquor and dispersant; Solid line: mother liquor and dispersant with ultrasounds (for experimental details see text). 
stress that the best dispersion effect is obtained by using the two techniques simultaneously.

\section{Conclusions}

The influence exerted by several synthesis parameters on the morphology, size and agglomeration of magnesium hydroxide particles precipitated in dilute aqueous solution was studied. It has been shown that the chemical nature of the base precipitant is of prime importance: the use of sodium hydroxide at $60^{\circ} \mathrm{C}$ leads to the surprising globular cauliflower-like agglomerates, while synthesis driven with aqueous ammonia promotes the obtaining of platelet-shaped particles. This is attributed to both the influences of $\mathrm{pH}$ and of cation structure in solution. The temperature also has a strong effect, mainly on the agglomeration behaviour and size of the particles. The particles show a tendency towards intergrowth at $60^{\circ} \mathrm{C}$, while at lower temperature, single and circular platelets are obtained, with a mean diameter depending on the synthesis and ageing temperature: the lower the temperature, the higher the diameter. Varying the magnesium source modifies the chemical nature of counter-ions in solution. Magnesium nitrate and magnesium chloride both gives rise to the desired morphology. Magnesium sulphate appears to promote agglomeration of primary nuclei, but this behaviour is not totally understood. Submitting the powders in solution to a mild hydrothermal treatment induces a pronounced improvement of the particles morphology, as well as an increase of their mean size, with subsequent decrease of their specific surface area. Finally, good dispersion of the powders in aqueous suspension was obtained by performing ultrasonication on the solution containing small quantities of a commercially available anionic dispersant, Dispex N40V.

\section{Acknowledgements}

Dr. Miguel Angel Rodriguez from the Institute for Ceramics and Glass (ICV-CSIC), Madrid, Spain, is greatly acknowledged for specific surface area measurements and valuable scientific discussion.

\section{References}

[1] R.J. Hall, D.R.F. Spencer, Interceram. NR3 (1973) 2112.

[2] F.H. Norton, Refractories, 4th Edition, McGraw-Hill, New York 1968, p. 80.

[3] R.N. Rothon, Magnesium Hydroxide: New Products, Processes and Applications, Intertech, 19 Northbrook Drive, Portland, Maine (Ed.), Functional Effect Filler, Berlin, Germany, 2000.

[4] Flamemag International Cie, State of the art: Magnesium Hydroxide, 2000.

[5] R.N. Rothon, D. Carus, D. Elsner, D. McCallum, Magnesium Hydroxide as a Flame retardant Filler for Plastics, Chem. Aust. (Jan.-Feb.), 1999, 35.

[6] A. Durin-France, L. Ferry, J.M. Lopez Cuesta, A. Crespy, Polym. Int. 49 (2000) 1101.

[7] R.N. Rothon, P.R. Hornsby, Polym. Degrad. Stab. 54 (1996) 383.

[8] J.E. Gardolinski, L.C.M. Carrera, M.P. Cantao, F. Wypych, J. Mater. Sci. 35 (2000) 3113.

[9] V. Mehrotra, P. Giannelis, Solid State Commun. 77 (1991) 155.

[10] M.S. Wang, T.J. Pinnavaia, Chem. Mater. 6 (1994) 468.

[11] P.B. Messersmith, E.P. Giannelis, Chem. Mater. 6 (1994) 1719.

[12] R.A. Vaia, S. Vasudevan, W. Krawiec, L.G. Scanlon, E.P. Giannelis, Adv. Mater. 7 (1995) 154.

[13] M. Alexandre, G. Beyer, C. Henrist, R. Cloots, A. Rulmont, R. Jérôme, Ph. Dubois, Chem. Mater. 13 (2001) 3830 .

[14] M. Alexandre, G. Beyer, C. Henrist, R. Cloots, A. Rulmont, R. Jérôme, Ph. Dubois, Macromol. Rapid Commun. 22 (2001) 643.

[15] R. Krishnamoorti, K. Yurekli, Curr. Opin. Coll. Interface Sci. 6 (5-6) (2001) 464.

[16] M.J. Solomon, A.S. Almusallam, K.F. Seefeldt, A. Somwangthanaroj, P. Varadan, Macromolecules 34 (6) (2001) 1864.

[17] P. Pascal, Nouveau traité de Chimie Minérale, Masson et Cie, Paris, 1958.

[18] Mellor, J.W., A Comprehensive Treatise on Inorganic and Theoretical Chemistry, Longmans, Green \& Co LTD, Great Britain, 1929.

[19] L. Hackspill, J. Besson, A. Hérold, Chimie Minérale, Presses Universitaires de France, Paris, 1968.

[20] L. Desgranges, G. Calvarin, G. Chevrier, Acta Cryst. B 52 (1996) 82.

[21] H.R. Oswald, R. Asper, Bivalent metal Hydroxides, in R.M.A. Lieth (Ed.), Physics and Chemistry of Mineral with Layered Structures, Vol. 1, Reidel, Dordrecht, 1977, pp. 71-86.

[22] M. Laska, J. Valtyni, P. Fellner, Cryst. Res. Technol. 28 (7) (1993) 931. 
[23] W. Feitknecht, H. Braun, Helv. Chim. Acta 50 (1967) 2040.

[24] A. Packter, Cryst. Res. Tech. 20 (3) (1985) 329.

[25] M. Copperthwaite, N.H. Brett, Sci. Ceram. 8 (1976) 85.

[26] M. Laska, V. Khandl, P. Fellner, Chem. Papers-Chem Zvesti 48 (5) (1994) 313.

[27] T. Baird, P.S. Braterman, H.D. Cochrane, J. Crystal Growth 91 (1988) 610.

[28] US 3.787.558 to Steetley Ltd., 1974.

[29] US 5.872.169 to Flamemag International Gie, 1999.
[30] US 4.229.423 to Kaiser Aluminium \& Chemical Co., 1980.

[31] V.A. Phillips, J.L. Kolbe, H. Opperhauser, J. Crystal Growth 41 (1977) 228.

[32] T.E. Larson, A.M. Buswell, Ind. Eng. Chem. 32 (1) (1940) 132.

[33] H. Stephen, T. Stephen, Solubilities of Inorganic and Organic Compounds, Pergamon Press Ltd., England, 1963.

[34] La grande encyclopédie des minéraux, Gründ, 1987.

[35] J.P. Jolivet, M. Henry, J. Livage, De la solution à l'oxyde, Interéditions et CNRS Editions, Paris, 1994. 